\title{
Sur deux espèces d'Hémiptères Auchénorrhynques, nouvelles pour la Réunion et d'importance économique potentielle
}

\author{
Serge Quilici, Bernard Reynaud, Jacques Bonfils
}

\begin{abstract}
On two species new for Réunion Island (Mascarene) and of potential economical importance (Hemiptera, Auchenorrhyncha). During 1997, two species of Hemiptera Auchenorrhyncha Cicadomorpha new for Réunion Island have been collected, that could be of potential economic importance. Collection of Philaenus spumarius (L.) (Cercopidae, Aphrophorinae) adds a new superfamily and a new family to our knowledge of the island fauna. This polyphagous species can be noxious to various host plants and transmit Pierce Disease to vine crops. Nephotettix modulatus Melichar, 1912 (Cicadellidae, Deltocephalinae) belongs to a genus including various vectors of viruses on rice crops in Asia.
\end{abstract}

\section{Résumé}

Des récoltes effectuées en 1997 ont permis de déterminer deux espèces d'Auchénorrhynques Cicado-morpha nouvelles pour la Réunion et pouvant présenter une certaine nuisibilité pour les cultures. La récolte de Philaenus spumarius (L.) (Aphrophorinae) permet d'ajouter une nouvelle super-famille et une nouvelle famille à la faune connue de l'île. Cette espèce polyphage peut commettre des dégâts sur diverses plantes-hôtes et transmettre la maladie de Pierce à la vigne. Nephotettix modulatus Melichar, 1912 (Cicadellidae, Deltocephalinae) appartient à un genre comprenant divers vecteurs de viroses du riz en région asiatique.

\section{Citer ce document / Cite this document :}

Quilici Serge, Reynaud Bernard, Bonfils Jacques. Sur deux espèces d'Hémiptères Auchénorrhynques, nouvelles pour la Réunion et d'importance économique potentielle. In: Bulletin de la Société entomologique de France, volume 103 (4), octobre 1998. pp. 369-372;

https://www.persee.fr/doc/bsef_0037-928x_1998_num_103_4_17445

Fichier pdf généré le 25/09/2019 


\title{
Sur deux espèces d'Hémiptères Auchénorrhynques, nouvelles pour la Réunion et d'importance économique potentielle
}

\author{
par Serge QUILICI*, Bernard REYNAUD** et Jacques BONFILS*** \\ *CIRAD-FLHOR Réunion, station de Bassin-Martin, laboratoire d'Entomologie, B.P. 180, F - 97455 Saint-Pierre \\ cedex. Adel : quilici@cirad.fr. ${ }^{* *}$ CIRAD-AMIS Réunion, laboratoire 3-P, 7 chemin de l'IRAT, F - 97410 Saint- \\ Pierre cedex. Adel : reynaud@cirad.fr. ***10 rue des Bouvreuils, Aiguefonde, F - 81200 Mazamet
}

Résumé. - Des récoltes effectuées en 1997 ont permis de déterminer deux espèces d'Auchénorrhynques Cicadomorpha nouvelles pour la Réunion et pouvant présenter une certaine nuisibilité pour les cultures. La récolte de Philaenus spumarius (L.) (Aphrophorinae) permet d'ajouter une nouvelle super-famille et une nouvelle famille à la faune connue de l'île. Cette espèce polyphage peut commettre des dégâts sur diverses planteshôtes et transmettre la maladie de Pierce à la vigne. Nephotettix modulatus Melichar, 1912 (Cicadellidae, Deltocephalinae) appartient à un genre comprenant divers vecteurs de viroses du riz en région asiatique.

Summary. - On two species new for Réunion Island (Mascarene) and of potential economical importance (Hemiptera, Auchenorrhyncha). During 1997, two species of Hemiptera Auchenorrhyncha Cicadomorpha new for Réunion Island have been collected, that could be of potential economic importance. Collection of Philaenus spumarius (L.) (Cercopidae, Aphrophorinae) adds a new super-family and a new family to our knowledge of the island fauna. This polyphagous species can be noxions to various host plants and transmit Pierce Disease to vine crops. Nephotettix modulatus Melichar, 1912 (Cicadellidae, Deltocephalinae) belongs to a genus including various vectors of viruses on rice crops in Asia.

Key-words. - Hemiptera, Homoptera, Cicadomorpha, Cercopidae, Aphrophorinae, Cicadellidae, Deltocephalinae, Réunion island, Philaenus spumarius, Nephotettix modulatus, economic importance.

La faune des Hémiptères Auchénorrhynques de l'île de la Réunion a fait l'objet, il y a quelques années, d'une première synthèse, récemment complétée (BONFILS et al., 1994, ATTIÉ et al., 1998).

Au cours de l'année 1997, deux nouvelles espèces, d'importance économique potentielle, ont été détectées dans cette île de l'océan Indien occidental. La collecte de Philaenus spumarius (L.) (Cercopidae, Aphrophorinae) permet d'ajouter une nouvelle super-famille et une nouvelle famille à la faune connue de l'île. Cette espèce, à large répartition paléarctique et néarctique, n'avait janiais été détectée jusqu'alors en zone tropicale insulaire. Polyphage, elle est susceptible de commettre des dégâts sur diverses plantes et de transmettre des pathogènes sur certaines d'entre elles.

La collecte de Nephotettix modulatus Melichar, 1912 constitue la première mention de ce genre dans l'île et porte à 19 le nombre d'espèces de Cicadellidae Deltocephalinae connues dans l'île. Cette espèce africaine appartient à un genre comprenant diverses espèces vectrices de viroses, notamment au riz dans la région asiatique. A la Réunion, en l'absence de production rizicole, $N$. modulatus a été collecté uniquement sur Paspalidium geminatum (Forssk.) Stapf, en zone humide.

Philaenus spumarius (Linné, 1758)

$=$ Philaenus leucophtalmus $(\mathrm{L}$.)

L'Aphrophore écumeuse ("Common spittle bug" ol! "Meadow spittle bug" des anglo-saxons) est un Cercopide polyphage très abondant en Europe, Asie, Japon et Amérique du Nord. 
Dans son aire habituelle de distribution, les adultes présentent une faible parapause, induite par la photopériode: les femelles qui apparaissent en mai-juin n'effectuent leur ponte qu'en septembre-octobre. Il existe une diapause embryonnaire obligatoire chez cette espèce, qui est levée par le froid hivernal (MÜLLER, 1979), l'éclosion des œufs ayant lieu en mai. Pendant tout leur développement, les larves se recouvrent d'une masse spumeuse, avant d'atteindre leur maturité au cours de l'été (ALFORD, 1991).

En Amérique du Nord, $P$. spumarius est le Cercopidae le plus abondant, le plus répandu et celui qui présente la plus grande importance économique. Généralement abondant sur de nombreux arbres, arbustes et plantes basses, c'est un ravageur mineur de la lavande (Lavandula) et de divers autres végétaux d'ornement: Aster, Berberis, Campanula, Chrysanthemum, Coreopsis, Geum (benoîte), Lychnis, Mahonia, Phlox, Rosa (rosier), Rudbeckia, Solidago, etc. (ALFORD, 1991).

Les jeunes pousses des plantes sensibles, infestées par les larves, peuvent se déformer et flétrir; dans certains cas, les fleurs sont malformées. Les adultes ne causent aucun dégât évident. Par ailleurs, les végétaux d'ornement sont déparés par la présence des "crachats de coucou", ce qui peut réduire la valeur marchande des plantes cultivées en conteneurs dans les pépinières (ALFORD, 1991).

$P$. spumarius est connu pour transmettre divers agents pathogènes dans la région néarctique (BRCAK, 1979). Ainsi, en Amérique du Nord, c'est un vecteur avéré du Pierce Disease (PD)à la vigne et de l'Almond Leaf Scorch (ALS) à l'amandier (PURCELL, 1979, 1980), dont l'agent causal est la bactérie Xylella fastidiosa (PURCELL \& HOPKINS, 1996).

Matériel étudié: sur fraisier, Petit-Tampon (alt. $850 \mathrm{~m}$ ) (réf.: RQ 3397; C. Chane-To)

A la Réunion, l'espèce n'a pour l'instant été détectée, aux stades larvaire et adulte, qu'en une seule occasion chez un producteur de fraisiers du sud de l'île, dont l'exploitation est située à $850 \mathrm{~m}$ d'altitude. D'autres prospections ultérieures n'ont pas permis de la retrouver. Il est possible que $P$. spumarius ait atteint la Réunion au stade œuf, sur des plants de fraisiers importés. Il n'est pas certain que cette espèce à diapause puisse trouver dans les conditions des Hauts de la Réunion les conditions écologiques favorables à un établissement permanent. Le climat tropical des bas de l'île devrait en outre lui être défavorable.

D'un point de vue agronomique, le devenir de l'espèce dans l'île est à surveiller. Outre d'éventuels dégâts directs sur diverses plantes-hôtes, on pourrait craindre en effet sa capacité de transmettre des pathogènes aux cultures de vigne, qui sont situées dans les bas de l'île (raisin de table) mais aussi en zone d'altitude (raisin de cuve dans le cirque de Cilaos).

Nephotettix modulatus Melichar, 1912

Le genre Nephotettix Matsumura, l'un des plus important genres de vecteurs de la zone orientale, a été revu par GHAURI (1971); il comprend 8 espèces. N. modulatus est présent dans de nombreux pays d'Afrique, à l'exception du sud du continent (WILSON \& CLARIDGE, 1991). Au contraire, diverses autres espèces de Nephotettix ont une distribution asiatique. 
Depuis les années 70, le "tungro" est la maladie virale la plus grave sur riz en Asie du Sud et du Sud-Est. Cette maladie est due à l'infection conjointe de deux virus: le rice tungro bacilliform virus (RTBV) et le rice tungro spherical virus (RTSV). Ces virus sont transmis par Recilia dorsalis (Motschulsky) et cinq espèces de Nephotettix: $N$. virescens (Distant), N. nigropictus (Stål), N. cincticeps Uhler, N. malayanus Ishihara $\&$ Kawase et $N$. parvus Ishihara \& Kawase (KHAN et al., 1991). En outre, plusieurs autres viroses du riz sont transmises en Asie par des Nephotettix spp. (NAULT, 1987). Selon INOUE (1983), la gamme de plantes-hôtes du genre est restreinte aux Poacées; seul AKINGBOHUNGBE (1983) signale la récolte d'une série de spécimens de $N$. modulatus sur coton au Nigéria.

Matériel étudié: nombreux ô et $q$, étang de Saint-Paul, environ de St-Paul, 5.III.1997; au filet fauchoir et au D-Vac (réf.: RR 62 et RR63; B. Reynaud et M. Grondin.); 14 $\delta, 13 \%$, étang du Gol, environ de Saint-Louis 21.II.1998; au filet fauchoir (réf.: RR $111 ;$ B. Reynaud.).

A la Réunion, cette espèce a été d'abord récoltée dans le nord-ouest de l'île, dans la zone humide de l'étang de Saint-Paul, puis aux abords de l'étang du Gol, autre zone humide située dans le sud-ouest de l'île. Elle semble présente uniquement sur une Poacée pérenne de distribution pantropicale, Paspalidium geminatum (Forssk.) Stapf, présente également à l'île Maurice, à Madagascar et en Afrique de l'Est (BOSSER, 1969). Cette Cicadelle est très abondante lorsque $P$. geminatum se développe en zone inondée (environ 500 individus par $\mathrm{m}^{2}$ ) et beaucoup plus rare lorsque la zone se dessèche (environ 10 individus par $\mathrm{m}^{2}$ ) (LIN TANFF \& BÉNARD, 1997). Avant même sa détermination, GUILLERMET \& COUTEYEN (1996) avaient utilisé son abondance comme indicateur biologique de la qualité des milieux dans la zone de l'étang de Saint-Paul. $P$. geminatum est présente dans les plaines alluviales côtières de l'ouest, du sud et du nord-est. Il faudrait donc vérifier la présence de $N$. modulatus dans le nord-est de l'île.

WILSON \& CLARIDGE (1991) signalaient l'absence de plante-hôte connue de $N$. modulatus en Afrique; nos résultats montrent qu'au moins à la Réunion, cette espèce est inféodée à la Poacée $P$. geminatum. Par ailleurs, le signalement de cette Cicadelle à la Réunion permet d'étendre vers l'est l'aire de répartition connue de l'espèce.

REMERCIEMENTS. - Les auteurs tiennent à remercier vivement C. Chane-To (ARMEFLHOR: Association Réunionnaise pour la Modernisation de l'Economie Fruitière, Légumière et Horticole), pour la transmission de l'échantillon de P. spumarius, ainsi que Chr. Guillermet, directeur de l'Insectarium de la Réunion, qui nous a permis de faire état de ses données sur $N$. modulatus.

\section{AUTEURS CITÉS}

AKINGBOHUNGBE A.E., 1983. - Nomenclatural problems, biology, host plant and possible vector status of Auchenorrhyncha associated with crop plants in Nigeria, p. 365-370. In: W.J. Knight, N.C. Pant, T.S. Robertson \& M.R. Wilson (eds), Ist International Workshop on Leafhoppers and Planthoppers of Economic Significance. CAB / CIE, 500 p.

ALFORD D.V., 1991. - A colour atlas of pests of ornamental trees, shrubs and flowers. Manson Publ. Ltd, London, UK, $464 \mathrm{p}$.

ATTIÉ M., BONFILS J. \& QUILICI S., 1998. - Hémiptères Auchénorrhynques nouveaux pour la faune de

l'île de la Réunion. Bulletin de la Société entomologique de France, 103 (3) : 255-262.

Bonfils J., Quilici S. \& ReYNAud B., 1994. - Les Hémiptères Auchénorrhynques de l'île de la 
Réunion. Bulletin de la Société entomologique de France, 99 (3): 227-240.

BOSSER J., 1969. - Graminées des pâturages et des cultures à Madagascar. Mémoires ORSTOM, 35, $440 \mathrm{p}$.

BRCAK J., 1979. - Leafhopper and planthopper vectors of plant disease agents in central and southern

Europe. In: Leafhoppers vectors and plant disease agents. K. Maramorosch \& K.F. Harris (eds). Academic Press Inc, New-York, 654 p.: 97-154.

GHAURI M.S.K., 1971. - Revision of the genus Nephotettix Matsumura (Homoptera: Cicadelloidea:

Euscellidae) based on the type material. Bulletin of entomological Research, 6: 481-512.

GUILleRMET C. \& COUTEYEN S., 1996. - Inventaire partiel de l'entomofaune de l'Etang de Saint-

Paul. Etude d'impact pour le Conseil régional de la Réunion, $80 \mathrm{p}$.

INOUE H., 1983. - Reproductive isolation mechanisms and hybridization of Nephotettix spp. In: W.J.

Knight, N.C. Pant, T.S. Robertson \& M.R. Wilson (eds), lst International Workshop on Leafhoppers and Planthoppers of Economic Importance. CAB / CIE, 500 p. : p. 339-349.

Khan M. A., Hibino H., AgUiero V. M., PaQuiOAg R.D. \& OPINA O.S., 1991. - Rice and weed hosts of rice tungro-associated viruses and leafhoppers vectors. Plant Disease, 75: 926-930.

LIN TANFF A. \& BÉNARD S., 1997. - L'Etang de Saint-Paul: étude de la communauté d'arthropodes associés à une prairie de graminées. Mémoire de Licence de Biologie, Univ. de la Réunion, 37 p.

MÜLLER H.J., 1979. - Effects of photoperiod and temperature on leafhopper vectors. In: Leafhoppers vectors and plant disease agents. K. Maramorosch \& K.F. Harris (eds). Academic Press Inc, NewYork, 654 p.: 29-94.

NAULT L.R., 1987. - Origin and evolution of Auchenorrhyncha-transmitted, plant infecting viruses. In: M.R. Wilson \& L.R. Nault (eds), Proc. 2nd Int. Workshop on Leafhoppers and Planthoppers of Economic Importance, held Provo, Utah, USA, 28th July-1st August 1986. CIE, London, 368 p. : 131-149.

PURCELL A.H., 1979. - Leafhopper vectors of xylem-borne plant pathogens. In: Leafhoppers vectors and plant disease agents. K. Maramorosch \& K.F. Harris (eds). Academic Press Inc, New-York, 654 p., chap. 19: 603-625.

1980. - Almond Leaf Scorch: leafhopper and spittlebug vectors. Journal of economic Entomology, 73 (6) : 834-838.

PIJRCFI.I. A.H. \& HOPKINS T. L., 1996. - Pathogens: bacteria and other prokariotes. Fastidious xylemlimited bacterial plant pathogens. Annual Review of Phytopathology, 34: 131-152.

WILSON M. R. \& CLARIDGE M. F., 1991. - Handbook for the identification of leafhoppers and planthoppers of rice. $\mathrm{CAB}$ International/NRI, $142 \mathrm{p}$.

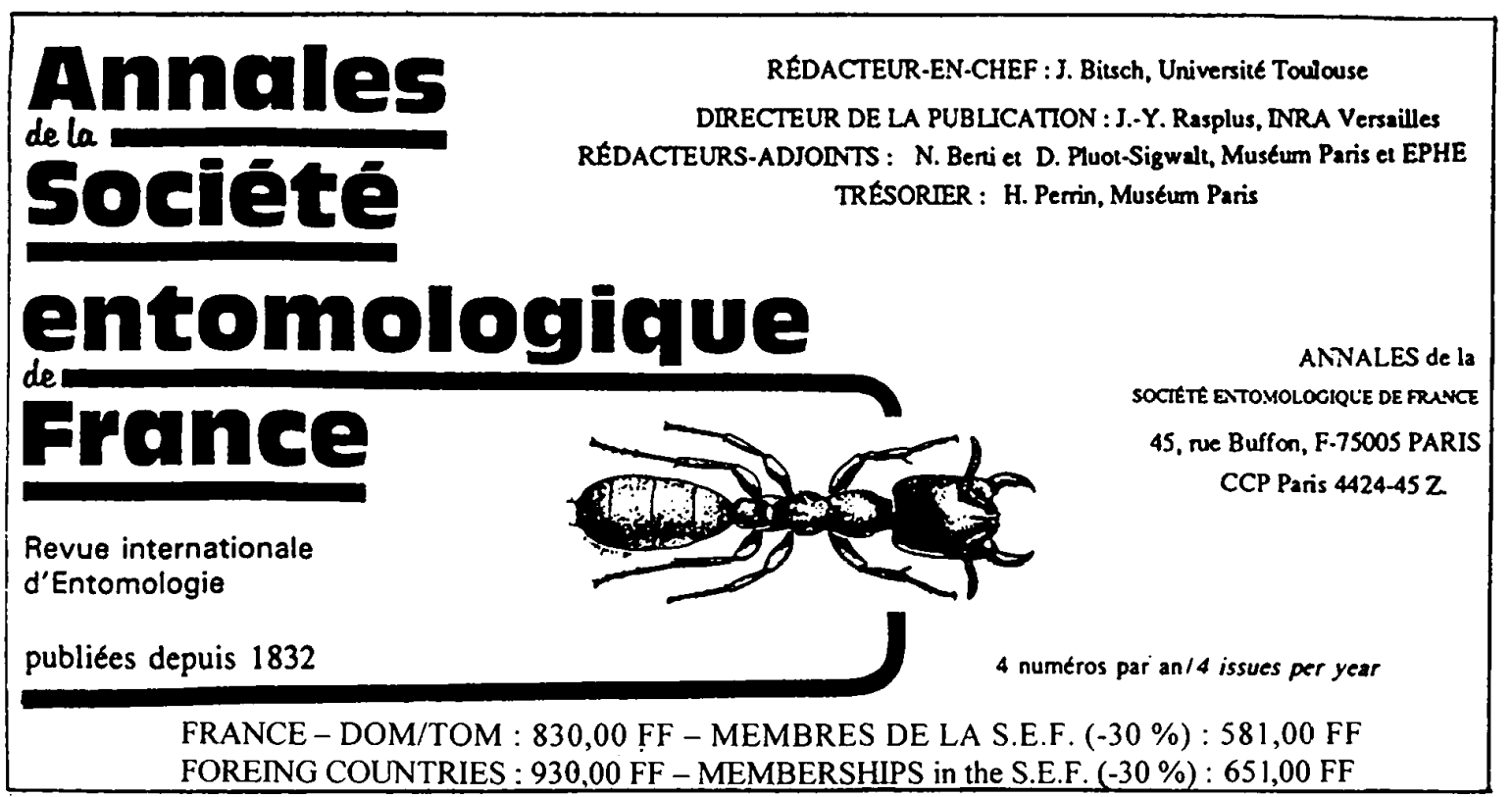

